\title{
CLOUD CAVITATION DYNAMICS
}

\author{
MILES WILSON ${ }^{1}$, JOHN R. BLAKE ${ }^{\otimes} 1$ and PETER M. HAESE ${ }^{1}$
}

(Received 26 September, 2008)

\begin{abstract}
An analysis is developed for the behaviour of a cloud of cavitation bubbles during both the growth and collapse phases. The theory is based on a multipole method exploiting a modified variational principle developed by Miles ["Nonlinear surface waves in closed basins", J. Fluid Mech. 75 (1976) 418-448] for water waves. Calculations record that bubbles grow approximately spherically, but that a staggered collapse ensues, with the outermost bubbles in the cloud collapsing first of all, leading to a cascade of bubble collapses with very high pressures developed near the cloud centroid. A more complex phenomenon occurs for bubbles of variable radius with local zones of collapse, with a complex frequency spectrum associated with each individual bubble, leading to both local and global collective behaviour.
\end{abstract}

2000 Mathematics subject classification: primary 76T10; secondary 76B10. Keywords and phrases: bubbles, multipoles, clouds, clusters, cavitation.

\section{Introduction}

Understanding the motion and deformation of bubbles within three-dimensional flows is of prime importance to a large number of engineering applications, such as cavitation erosion, chemical and biological reactors, and underwater explosion bubbles. The flows occur when the bubbles are subjected to a variable pressure field, as occurs during hydraulic cavitation, and depending on the form of the initial surface disturbance, a variety of physical and parametric instabilities may arise. In one case the bubble surfaces exhibit progressively larger deformations from spherical, resulting from a transference of kinetic energy from the breathing mode (Rayleigh mode) to higher ones; a second instability results in an erratic dancing motion similar to Brownian motion.

Force balance equations for the rate of change of the Kelvin impulse have often been exploited to develop approximate dynamical models for the motion of a few bubbles. In the Lagally formulation of the multipole method (see Taylor [13], Landweber and

\footnotetext{
${ }^{1}$ School of Mathematics, University of Birmingham, Edgbaston, Birmingham B15 2TT, UK; e-mail: j.r.blake@bham.ac.uk.

(C) Australian Mathematical Society 2009, Serial-fee code 1446-1811/2009 \$16.00
} 
Yih [9], and Landweber and Miloh [7, 8] for further details), this leads to a particularly simple expression relating the rate of change of the Kelvin impulse to the singularities of the analytically continued potential flow. If the potential solution is known or can be calculated, then this expression results in an extremely effective method of calculating a bubble's evolution.

In past studies, Lagally's theorem has been extremely successful at predicting the dynamics of a few bubbles situated within various flow geometries. For example, Kucera and Blake [6] used a source-dipole representation of the bubble to model its growth and collapse near complex rigid boundaries. Their results indicated that such a model may be generally at most $3 \%$ in error, for such quantities as the bubble volume, Kelvin impulse, and bubble trajectory toward the boundary. Higher-order approximations were used by Best and Blake [1], again to obtain criteria for the migration of the bubble.

For many engineering applications, it is of prime importance to understand and predict the often violent surface dynamics of a bubble assembly. In this paper, therefore, we turn our attention to the growth and collapse dynamics of moderately sized bubble clouds. Such a situation will occur if the bubbles are suddenly subjected to a rapid changes in pressure within the ambient fluid. Of particular interest is the collapse phase of a cloud of cavitation bubbles, initiating first at the outer bubbles and proceeding inwards towards the array's "centre"; an example of this was briefly reported by Blake et al. [2]. It was Hanson et al. [4] who speculated that such a collapse is the result of the inward propagation of a "shock" towards the cloud's "centre", and enhances the potential for both noise and erosion. Further enhancement occurs by fluid mechanical coupling of the individual bubble's motion, through the pressure and velocity field.

In this paper we first specify the problem in terms of spatial and surface modal functions, exploiting Miles' variational approach by requiring the kinetic energy to be stationary with respect to variations in $\phi$; the resulting Lagrangian is independent of the generalized momenta. A weighted residual formulation is applied in conjunction with Bernoulli's equation and continuity of the surface velocities. This results in a coupled set of differential equations for the rate of change of the velocity potential, and we use these in an algorithm to step the bubble surfaces forward with time for a selection of examples for bubbles in both a regular array and more randomly distributed in both location and initial bubble radius.

\section{Formulation of equations of motion}

We consider an unbounded, irrotational, incompressible flow that is inviscid at large Reynolds numbers. The flow satisfies Laplace's equation

$$
\nabla^{2} \phi=0
$$

where $\nabla^{2}$ is the Laplacian operator and $\phi$ is the velocity potential of the flow. The fluid velocity $\mathbf{u}$ is specified by the gradient of the velocity potential. We demand a 
no radiation condition at infinity so that $\phi=O\left(r^{-1}\right)$ for large $r$, and the boundary condition

$$
\frac{\partial \phi}{\partial n}=\mathbf{u}_{i} . \mathbf{n}
$$

at the surface of all bubbles, where $\mathbf{n}$ is the unit outward normal vector to the surface, and $\mathbf{u}_{i}$ the surface velocity of bubble $i\left(i=1, \ldots, n_{b}\right)$. The kinematic boundary condition is thus uniquely determined. This analysis follows the development for clouds of bubbles of constant volume by Wilson et al. [15], but has a different farfield condition allowing volume change associated with the collective source-sink behaviour of the bubble cloud.

To solve the specified boundary value problem we follow Miles [11] and represent the potential $\phi$ throughout the flow by a set of time-dependent coefficients $\phi^{k}$ and spatial functions $\chi_{k}$. These quantities are related by

$$
\phi=\phi^{k} \chi_{k}
$$

We do the same for the normal surface velocity of each bubble $i$, so

$$
\mathbf{u}_{i} . \mathbf{n}=U_{i}^{k} \psi_{k},
$$

where $\psi_{k}$ is a surface modal function, and $U_{i}^{k}$ its corresponding time-dependent amplitude.

The boundaries of the fluid are the $n_{b}$ bubble surfaces. We denote the complete boundary by $\partial S$ and the individual bubble surfaces by $S_{i}$. Following Miles [11], Wilson et al. [15] and Wilson [14], the total fluid kinetic energy can be expressed as

$$
\mathcal{T}=-\frac{1}{2} \rho \int_{\partial S} \phi \frac{\partial \phi}{\partial n} d S=\frac{1}{2} \rho \int_{\partial S} \phi \frac{\partial \phi}{\partial n} d S-\rho \sum_{i=1}^{n_{b}} \int_{S_{i}} \phi \mathbf{u}_{i} . \mathbf{n} d S .
$$

Obtaining this relationship exploits the zero contribution to the kinetic energy from the spherical control surface, $S_{\infty}$, at infinity and by breaking the expression up into a combination of variation in $\phi$ and applying the boundary conditions on the bubble surface. This can be simplified using (2.1) and (2.2) to give

$$
\mathcal{T}=\frac{1}{2} \rho \boldsymbol{\Phi}^{T} \mathbf{k} \boldsymbol{\Phi}-\rho \boldsymbol{\Phi}^{T} \mathbf{d} \mathbf{U},
$$

where $\boldsymbol{\Phi}$ and $\mathbf{U}$ are column matrices composed of the coefficients $\phi^{k}$ and $U_{i}^{k}$ respectively, and the entries of the symmetric square matrix $\mathbf{k}$ and rectangular matrix $\mathbf{d}$ are

$$
k_{j k}=\int_{\partial S} \chi_{j} \frac{\partial \chi_{k}}{\partial n} d S \text { and } d_{j k}=\int_{S_{i}} \chi_{j} \psi_{k} d S .
$$

Miles proposed that the boundary value problem should be determined by requiring the kinetic energy to be stationary with respect to a small variation $\delta \phi$, so

$$
\frac{\partial}{\partial \boldsymbol{\Phi}}\left[\frac{1}{2} \rho \boldsymbol{\Phi}^{T} \mathbf{k} \boldsymbol{\Phi}-\rho \boldsymbol{\Phi}^{T} \mathbf{d} \mathbf{U}\right]=\mathbf{0} .
$$


In the context of classical mechanics, the coefficients $\phi^{k}$ are the generalized momenta of the system, and the variational requirement of Miles follows the classical mechanics result that the Lagrangian is independent of these generalized momenta.

Using (2.3) we find

$$
\mathbf{k} \boldsymbol{\Phi}=\mathbf{d U},
$$

which may be inverted to yield the multipole solution $\boldsymbol{\Phi}$ if the components of the surface velocities $\mathbf{U}$ are given. However, for our study here we wish to determine the surface velocities and hence surface evolution. Thus we need to determine the rate of change of $\Phi$ which is to be developed in the next section.

\section{Bubble deformation}

We now outline a method to derive $\dot{\phi}$ by exploiting the dynamic boundary conditions on the bubble surface.

Miles [11], who in turn refers to Luke [10], noted that a Lagrangian can be constructed for water waves which provides an integral equation of the form

$$
\int\left(p-p_{a}\right) \mathcal{W}(r) d S=0 .
$$

The integral is taken over the water surface, and $p_{a}$ is the atmospheric pressure. Substitution of (2.1) yields a coupled set of first-order differential equations in $\dot{\phi}$. This argument cannot be directly extended and needs to be modified slightly for bubbly flows since the internal bubble dynamics must be coupled and the surface integral would be infinite in the far field. The method exploited below is an adapted weighted residual method.

At the free surface of bubble $i$, continuity of the internal pressure $p_{i}$ and fluid pressure $p$ can be written using Bernoulli's equation

$$
\left[p_{i}-p_{\infty}-\rho\left(\dot{\phi}^{k} \chi_{k}+\phi^{k} \dot{\chi}_{k}\right)-\frac{1}{2} \rho\left|\phi^{k} \nabla \chi_{k}\right|^{2}\right]_{S_{i}}=0 .
$$

Multiplying by a weighting function $\mathcal{W}(\mathbf{r})$ and integrating over the collective flow boundaries $\partial S$ yields

$$
\rho \dot{\phi}^{k} \int_{\partial S} \mathcal{W}(\mathbf{r}) \chi_{k} d S=\sum_{i} \int_{S_{i}} \mathcal{W}(\mathbf{r})\left[p(\mathbf{r}, t)-p_{i}\right]_{\Phi} d S
$$

where the subscript denotes evaluation of the bracketed term at constant $\boldsymbol{\Phi}$. The choice of $\mathcal{W}=\partial \chi_{i} / \partial n$ for the weighting function was made so the equation can be written in the form

$$
\mathbf{k} \dot{\boldsymbol{\Phi}}=\mathbf{M}
$$

where $\mathbf{k}$ is the square matrix defined in (2.4) and $\mathbf{M}$ is a column matrix with components

$$
M_{j}=\sum_{i} \int_{S_{i}} \mathcal{W}(\mathbf{r})\left[p(\mathbf{r}, t)-p_{i}\right]_{\Phi} d S .
$$


In general the surface integrals are carried out numerically, and the coupled equations of motion (2.4) and (3.1) are solved concurrently using an Adam-MoultonBashforth/Runge-Kutta method.

The numerical algorithm proceeds as follows:

(1) At some initial moment in time the surface velocity is known or postulated. The components of $\mathbf{k}$ and $\mathbf{d}$ are calculated and initial amplitudes of the potential expansion coefficients in $\boldsymbol{\Phi}$ are determined.

(2) These coefficients are updated using Bernoulli's equation and continuity of pressure at the surface, through (3.1).

(3) With the potential solution known we use (2.4) to determine the surface velocity components, and integrate forward in time.

(4) Return to step (2).

\section{Calculation of the flow evolution in practice}

4.1. Potential functions The functions $\chi_{k}$ may be described by the expansions given in Hobson [5]. They have also been used by Sangani and Yao [12], who applied them to a fluid containing spherical bubbles situated randomly in space. They invoked periodicity in this domain by allowing a basic zone to repeat indefinitely, and hence formulated a solution throughout the entire fluid.

An array of otherwise isolated spherical bubbles can be represented by the multipole velocity potential

$$
\phi(\mathbf{r}, t)=\sum_{i=1}^{n_{b}} \sum_{n=0}^{\infty} \sum_{m=0}^{n}\left(A_{n m}^{i} \Delta_{m}+\tilde{A}_{n m}^{i} \tilde{\Delta}_{m}\right) \frac{\partial^{n-m}}{\partial x_{1}^{n-m}} \frac{1}{\left|\mathbf{r}-\mathbf{r}_{i}\right|}
$$

where $\mathbf{r}_{i}$ is the coordinate of the $i$ th bubble centroid. In practice we truncate this series appropriately. We define the differential operators $\left(\Delta_{m}, \tilde{\Delta}_{m}\right)$ in terms of the Cartesian coordinate system $\mathbf{r}=\left(x_{1}, x_{2}, x_{3}\right)$, in the complex forms

$$
\Delta_{m}=\left(\frac{\partial}{\partial \zeta}\right)^{m}+\left(\frac{\partial}{\partial \eta}\right)^{m} \text { and } \quad \tilde{\Delta}_{m}=\imath\left[\left(\frac{\partial}{\partial \zeta}\right)^{m}-\left(\frac{\partial}{\partial \eta}\right)^{m}\right]
$$

with $\zeta=x_{2}+\imath x_{3}, \eta=x_{2}-\imath x_{3}$.

By comparison with (2.1), we find that

$$
\phi_{k} \text { is replaced by }\left(A_{n m}^{i}, \tilde{A}_{n m}^{i}\right) \quad \text { and } \quad \chi_{k} \text { by }\left(\Delta_{m}, \tilde{\Delta}_{m}\right) \frac{\partial^{n-m}}{\partial x_{1}^{n-m}} \frac{1}{\left|\mathbf{r}-\mathbf{r}_{i}\right|} \text {. }
$$

Further details may be found in Wilson et al. [15].

4.2. Repositioning of the singularity coordinate If any part of a deformable surface approaches the singularity coordinate, the multipole scheme described will 
become unstable. To resolve beyond initial stages of deformation, therefore, the internal singularity is repositioned at the mean centroid position

$$
\overline{\mathbf{R}}_{i}=\frac{1}{\tau_{i}} \int_{S_{i}} \mathbf{R} d \tau,
$$

where $\tau_{i}$ is the bubble volume. The surface is described as

$$
\begin{gathered}
S_{i}=s_{i}-R_{i}=0, \\
R_{i}=\sum_{n=0}^{\infty} \sum_{m=0}^{n} a_{n m, i}^{\prime} Y_{n m}\left(\theta_{i}, \varpi_{i}\right)+\tilde{a}_{n m, i}^{\prime} \tilde{Y}_{n m}\left(\theta_{i}, \varpi_{i}\right),
\end{gathered}
$$

where $\left(Y_{n m}\left(\theta_{i}, \varpi_{i}\right), \tilde{Y}_{n m}\left(\theta_{i}, \varpi_{i}\right)\right)=P_{n}^{m}\left(\cos \theta_{i}\right)\left(\cos m \varpi_{i}, \sin m \varpi_{i}\right)$ are surface harmonics in a local spherical coordinate system fixed at the bubble centroid with radius $s_{i}$, azimuth angle $\varpi_{i}$, and polar angle $\theta_{i}$.

The surface coefficients $a_{n m, i}^{\prime}$ and $\tilde{a}_{n m, i}^{\prime}$ are calculated by considering various values of $\varpi_{i}$ and $\theta_{i}$, and expressing (4.2) in the over-described matrix form

$$
\mathbf{R}_{i}=\mathbf{Y}\left(\theta_{i}, \varpi_{i}\right) \mathbf{a}^{\prime}
$$

where $\mathbf{a}^{\prime} \equiv\left(a_{n m, i}^{\prime}, \tilde{a}_{n m, i}^{\prime}\right), \mathbf{Y}\left(\theta_{i}, \varpi_{i}\right) \equiv\left(Y_{n m}\left(\theta_{i}, \varpi_{i}\right), \tilde{Y}_{n m}\left(\theta_{i}, \varpi_{i}\right)\right)$, and $\mathbf{R}_{i} \equiv \mathbf{R}\left(\theta_{i}, \varpi_{i}\right)$. Inverting using the NAG routine E02GAF gives the new set of coefficients. The surface velocity components are evaluated in the same manner. Once the new surface description is evaluated, the code is restarted with a new initial value of the potential matrix $\boldsymbol{\Phi}$ using (3.1).

This algorithm can also provide a spectral analysis of the boundary integral method results (see below). During the computations an elemental description of the surface $\mathbf{R}_{i}$ is substituted into (4.3), and the corresponding amplitude of the spherical harmonic description is returned on inversion.

\section{Numerical simulations for the collapse of bubble arrays}

At the start of these simulations the bubbles' radii are set at a fraction $\eta$ of a typical radius, $R_{m}$. The internal gas pressure is then calculated from Rayleigh's equation such that $R_{m}$ is the maximum radius a bubble would achieve if isolated. The equations of motion are nondimensionalized on $R_{m}$, which is a usual method when studying the dynamics of cavitation and explosion bubbles. Generally for moderate size bubble arrays $(<12), \max (n)=5$ for both the potential and surface representation in expressions (4.1) and (4.2), corresponding in each case to 25 modes (or degrees of freedom) per bubble. For larger simulations, $\max (n)=3$.

For all of the simulations $\eta=0.2$ and $R_{m}=1.0 \mathrm{~mm}$, the corresponding internal gas pressure is $p_{g}=25.7 p_{\infty}$, which drives the initial growth. Based on bubble dynamics scalings, the Froude number (characterizing the effect of buoyancy) is $9.8 \times 10^{-5}$, which is negligible. The initial surface velocity, at $t=0$, is set to zero and the bubbles 


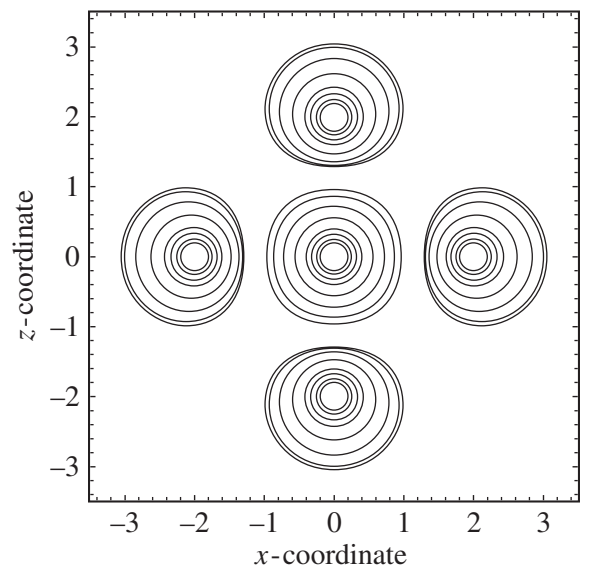

(a) Growth phase

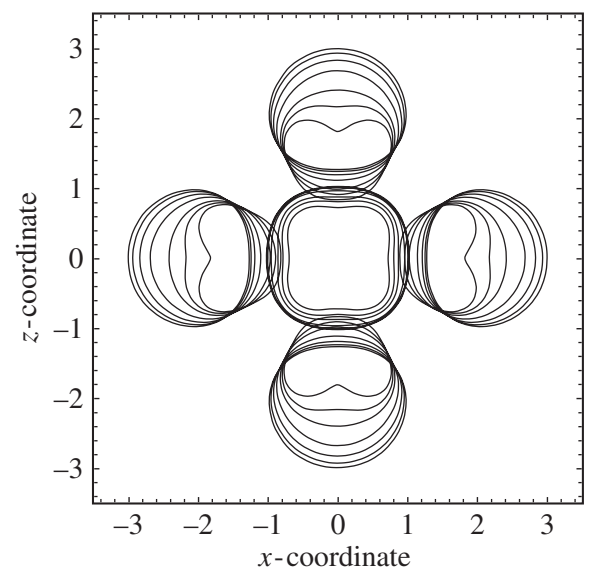

(b) Collapse phase

FIGURE 1. Growth and collapse phases for a five-bubble array having an initial radius $0.2 R_{m}=0.2 \times$ $10^{-4} \mathrm{~m}, p_{g}=25.7 p_{\infty}$.

are then allowed to expand due to the relatively high internal pressure. For such bubbles, we expect the effects of viscosity to play a inconsequential role on the surface dynamics.

Earlier results (Blake et al. [2]) in Figures 1 and 2 show the growth and collapse phases for compact square arrays of five and 13 bubbles, respectively. In each case the bubbles have initial size $\eta=0.2$. The bubbles' growth is almost spherical, though restricted on the surfaces closer to the centre. Contrary to what one might expect, the outer bubbles are not repelled away from the inner ones, but flatten off near the contact surfaces, surface tension being insufficient to retain spherical shape. Those at the corners are deformed slightly, owing to squeezing by the mid-side bubbles, which also exhibit some deformation. After about $2 \times 10^{-5} \mathrm{~s}$ the growth phase is complete, and the internal pressure reaches a minimum of about one fifth the far-field pressure. The collapse phase, driven by this relatively larger fluid pressure, is quite rapid and is characterized by the development of jets directed towards the central bubble, while translation of the body as a whole is minimal. The collapse then propagates from the outer bubbles inwards. During this stage the central bubble, having not quite attained a maximum radius, is to a large extent unaffected by this early stage of collapse. Towards the very last stages, there is a small degree of symmetry breaking, believed to be due to the inherent instability in the method as the jet comes through; note the right-hand and top bubbles. This cascading effect is further evident in Figure 3, which demonstrates the growth and collapse of a 20-bubble array positioned randomly.

We conclude with a simulation on irregular arrays with unequal radii, where an individual radius is chosen to vary randomly according to $a_{i}=\eta R_{m}(1+\zeta(i))$, and the internal pressure is chosen so as to achieve a maximum Rayleigh radius of 


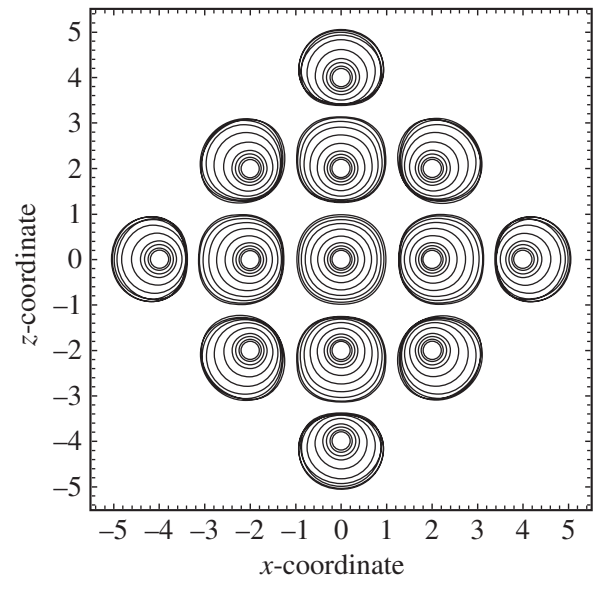

(a) Growth phase

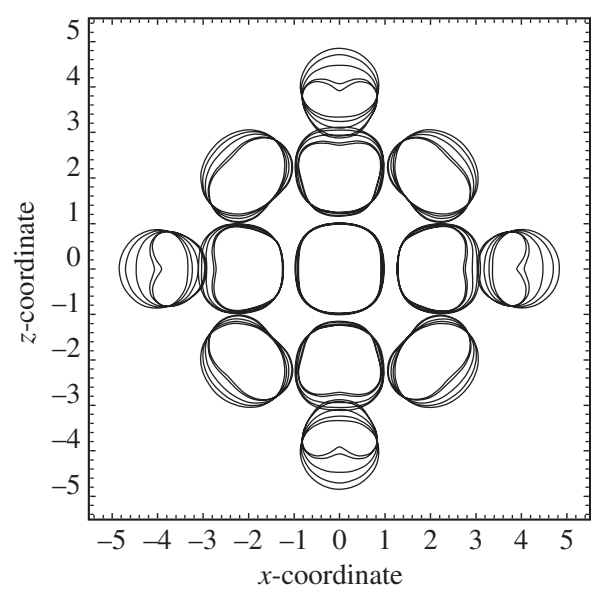

(b) Collapse phase

FIGURE 2. Growth and collapse phases for a 13-bubble array having an initial radius $0.2 R_{m}=0.2 \times$ $10^{-4} \mathrm{~m}, p_{g}=25.7 p_{\infty}$.

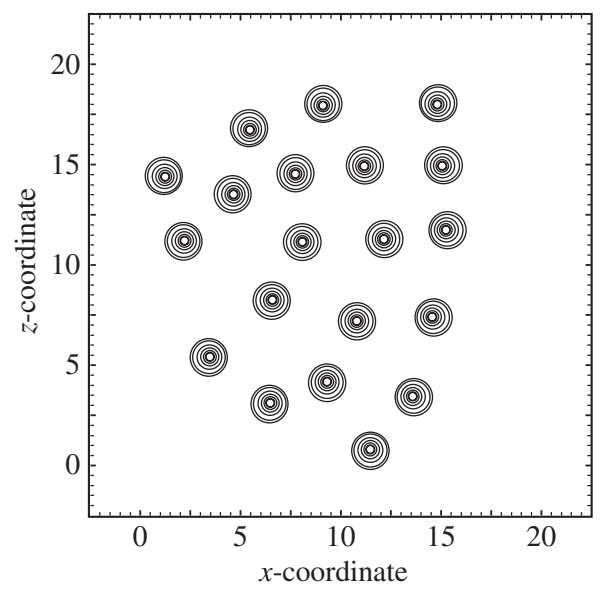

(a) Growth phase

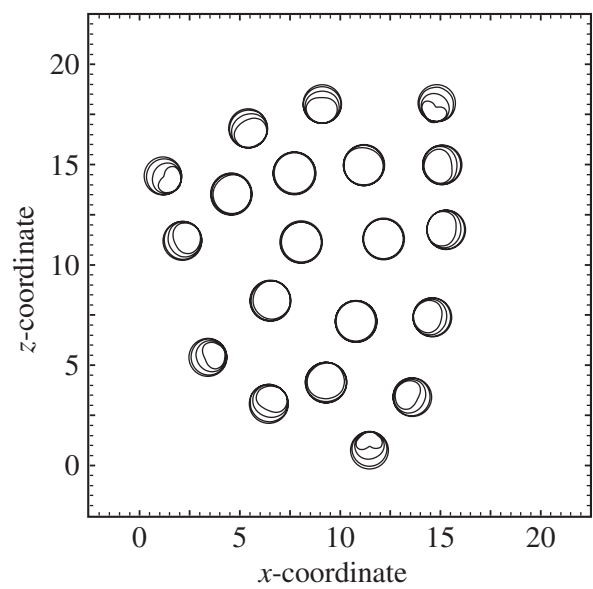

(b) Collapse phase

FIGURE 3. Growth and collapse phases for a 20-bubble array having an initial radius $0.2 R_{m}=0.2 \times$ $10^{-4} \mathrm{~m}, p_{g}=25.7 p_{\infty}$. 


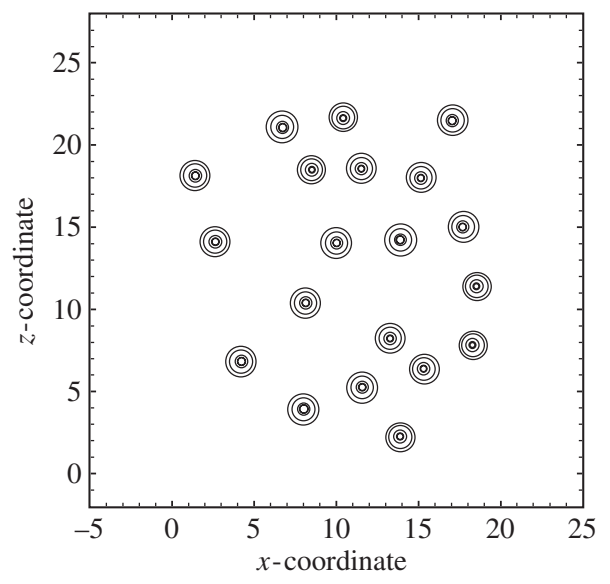

(a) Growth phase

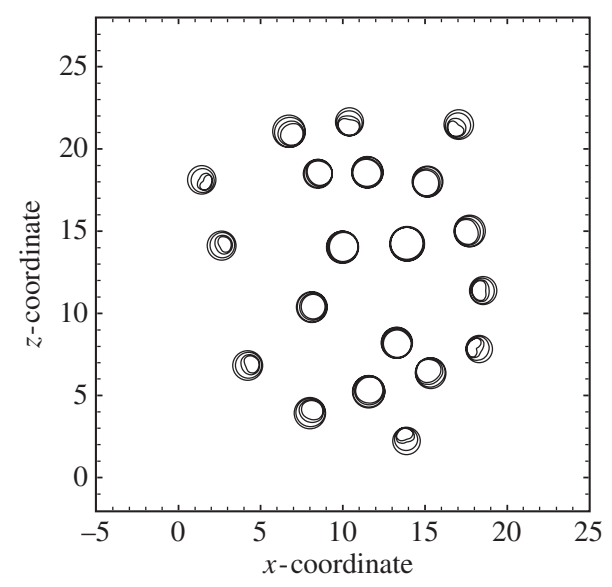

(b) Collapse phase

FIGURE 4. Growth and collapse phases for a 20-bubble array having an initial radius $0.2 R_{m}=0.2 \times$ $10^{-4}\left(1+\zeta_{i}\right) \mathrm{m}$, with $\zeta_{i}=(-0.3,0.3)$.

$a_{i}=\eta R_{m}(1+\zeta(i))$. Figure 4 demonstrates the variation in amplitude for a 20element array with $\zeta(i) \in(-0.3,0.3)$, and $R_{m}, \eta$ equal to $0.0001 \mathrm{~m}$ and 0.2 respectively. Again near-spherical growth develops, until about $2 \times 10^{-5} \mathrm{~s}$ when the smaller bubbles having attained their maximum volume first begin to collapse inwards, whilst the larger ones continue their growth. As before, jets first develop in the outer bubbles and are directed inwards towards the "cloud's" centre. Unfortunately the code halts after this first stage as these bubble calculations become numerically unstable. We expect, though, that this will initiate the arrays' total collapse. As $\zeta(i)$ increases in value there does not appear to be a well-defined layered collapse, since smaller bubbles now peak and collapse much earlier. It is expected in such a case that we would physically measure a number of distinct peaks in the pressure corresponding to each "cloud" collapse. A combined boundary integral-multipole method is currently under development to model the final stages of collapse of the cloud which will involve both toroidal and rebounding (smaller) bubbles.

\section{Conclusion}

We have concentrated on the collective collapse of a cloud of bubbles subjected to a variable pressure field including both a growth and collapse phase. These simulations highlight the fact that a "domino effect" operates during the collapse phase. For an assembly of bubbles of a similar size, the collapse propagates inwards through the fluid. The numerical results appear to predict the correct overall collapse behaviour up to the point where surface jets develop. There are, however, a number of details which it is unable to resolve. The most important is the resolution of the collapse beyond an initial jet profile. Since it is well known that the maximum fluid pressures occur just after the preliminary jet-surface impact, this is a region of much interest. 
Some of the results presented are preliminary but clearly reveal a range of applied problems that may be tackled using the method. Those specific to bubble-bubble interactions have been in excellent agreement with similar ones produced by other researchers, for example Chahine and Duraiswami [3]. However, the end of the bubbles' life is usually characterized by the formation of surface jets that pierce the main body (see, for example, [2]). Multipole schemes are inherently unstable because of the large number of terms required in such cases, and if results are required beyond the initial deformation stage a higher-order description for both the surface and potential is required. A combined boundary integral and multipole method is under development with the facility to model both the complex topology of an individual bubble and also the interaction with the other bubbles in the cloud.

\section{References}

[1] J. P. Best and J. R. Blake, "An estimate of the Kelvin impulse of a transient cavity", J. Fluid Mech. 261 (1994) 75-93.

[2] J. R. Blake, G. S. Keen, R. P. Tong and M. Wilson, "Acoustic cavitation: the fluid dynamics of non-spherical bubbles”, Philos. Trans. R. Soc. A357 (1999) 251-267.

[3] G. L. Chahine and R. Duraiswami, "Dynamical interactions in a multi-bubble cloud", J. Fluids Engrg. 114 (1992) 680-687.

[4] I. Hanson, V. K. Kendrinskii and K. A. Mørch, "On the dynamics of cavity clusters", J. Appl. Phys. 15 (1981) 1725-1734.

[5] E. W. Hobson, The theory of spherical and ellipsoidal harmonics (Cambridge University Press, Cambridge, 1931).

[6] A. Kucera and J. R. Blake, "Approximate methods for modelling the growth and collapse of cavitation bubbles near boundaries", Bull. Aust. Math. Soc. 41 (1990) 1-44.

[7] L. Landweber and T. Miloh, "Unsteady Lagally theorem for multipoles and deformable bodies", J. Fluid Mech. 96 (1980) 33-46.

[8] L. Landweber and T. Miloh, "Unsteady Lagally theorem for multipoles and deformable bodies. Corrigendum”, J. Fluid Mech. 112 (1981) 502.

[9] L. Landweber and C. S. Yih, "Forces, moments, and added masses for Rankine bodies", J. Fluid Mech. 1 (1956) 319-336.

[10] J. C. Luke, "A variational principle for a fluid with a free surface", J. Fluid Mech. 27 (1967) 395-397.

[11] J. W. Miles, "Nonlinear surface waves in closed basins", J. Fluid Mech. 75 (1976) 418-448.

[12] A. S. Sangani and C. Yao, "Bulk thermal conductivity of composites with spherical inclusions", J. Appl. Phys. 63 (1988) 1334-1341.

[13] G. I. Taylor, "The energy of a body moving in an infinite fluid, with application to airships", Proc. R. Soc. Lond. Ser. A 120 (1928) 13-21.

[14] M. Wilson, Mathematical modelling of bubble-vortex interactions, Ph. D. Thesis, University of Birmingham, 1997.

[15] M. Wilson, J. R. Blake and P. M. Haese, "A potential multipole theory for the hydrodynamics of bubble clouds", IMA J. Appl. Math. 73 (2008) 556-577. 